MADPH-95-903

UICHEP-TH/95-8

Rutherford Appleton Laboratory

RAL-TR-95-031

hep-ph/9507426

July 1995

\title{
Possible sneutrino-pair signatures with R-parity breaking
}

\author{
V. Barger ${ }^{a}$, W.-Y. Keung ${ }^{b}$ and R.J.N. Phillips ${ }^{c}$ \\ ${ }^{a}$ Physics Department, University of Wisconsin, Madison, WI 53706, USA \\ ${ }^{b}$ Physics Department, University of Illinois at Chicago, IL 60607-7059, USA \\ ${ }^{c}$ Rutherford Appleton Laboratory, Chilton, Didcot, Oxon OX11 OQX, UK
}

\begin{abstract}
If sneutrinos are the lightest supersymmetry partners and $R$-parity is not conserved, the process $e^{+} e^{-} \rightarrow \tilde{\bar{\nu}} \tilde{\nu}$ can have striking signatures due to the decay modes $\tilde{\nu} \rightarrow \ell^{+} \ell^{\prime-}$ or $\tilde{\nu} \rightarrow q \bar{q}^{\prime}$. We present cross section formulas and discuss event rates and detection at the upgraded $e^{+} e^{-}$collider LEP. Four-lepton signals should be detectable up to sneutrino mass $\tilde{m}_{\nu}=80 \mathrm{GeV}$ and maybe beyond; four-jet signals should be detectable up to $\tilde{m}_{\nu}=70 \mathrm{GeV}$, but would probably be obscured thereafter by $W W$ background.
\end{abstract}


Searches for supersymmetry (SUSY) particles depend considerably on the identity of the lightest SUSY partner (LSP), usually believed to be a neutralino[1]. Stable sneutrinos $\tilde{\nu}$ are strongly disfavored as LSP candidates, by a combination of constraints from $Z$ decay [2] that nowadays give mass $\tilde{m}_{\nu} \gtrsim M_{Z} / 2$ [3] and galactic dark-matter searches that together exclude the range $4 \mathrm{GeV}<\tilde{m}_{\nu}<1 \mathrm{TeV}$, 4 , 同. But in the presence of $R$-parity violation (RPV) [6,7,8,8, 10] the LSP can be unstable and sneutrinos are credible candidates once more; indeed the literature contains examples of SUSY-GUT parameter sets that lead to sneutrinos as LSP, either without[11] or with [12 RPV effects in the evolution equations. In the present paper we discuss and calculate the SUSY signals that will arise at an $e^{+} e^{-}$collider from the SUSY threshold process

$$
e^{+} e^{-} \rightarrow \tilde{\bar{\nu}}_{\alpha} \tilde{\nu}_{\alpha} \quad(\alpha=e, \mu, \tau)
$$

if approximately degenerate sneutrinos $\tilde{\nu}_{\alpha}$ are the LSP and decay by RPV processes. We ignore single-SUSY-particle production [7, 10], that can in principle take place with a lower threshold via RPV interactions, because the latter are either known [7,8] or suspected to have much much smaller couplings than the gauge couplings which control Eq.(1).

Sneutrino pair production proceeds via $t$-channel exchange of charginos $\chi_{i}^{ \pm}\left(\right.$for $e_{L}^{-} e_{R}^{+} \rightarrow$ $\tilde{\nu}_{e} \tilde{\bar{\nu}}_{e}$ only) and via $s$-channel $\mathrm{Z}$ exchange, that together give the helicity amplitudes

$$
\begin{aligned}
\mathcal{M}\left(e_{L}^{-} e_{R}^{+} \rightarrow \tilde{\nu}_{e} \tilde{\bar{\nu}}_{e}\right) & =\frac{e^{2} \beta s \sin \theta}{2 \sin ^{2} \theta_{W}}\left(\frac{\cos ^{2} \gamma_{R}}{t-m_{\chi_{1}^{+}}^{2}}+\frac{\sin ^{2} \gamma_{R}}{t-m_{\chi_{2}^{+}}^{2}}+\frac{-\frac{1}{2}+\sin ^{2} \theta_{W}}{\cos ^{2} \theta_{W}\left(s-M_{Z}^{2}\right)}\right), \\
\mathcal{M}\left(e_{L}^{-} e_{R}^{+} \rightarrow \tilde{\nu}_{\alpha} \tilde{\bar{\nu}}_{\alpha}\right) & =\frac{e^{2} \beta s \sin \theta}{2 \sin ^{2} \theta_{W}}\left(\frac{-\frac{1}{2}+\sin ^{2} \theta_{W}}{\cos ^{2} \theta_{W}\left(s-M_{Z}^{2}\right)}\right) \quad(\alpha=\mu, \tau), \\
\mathcal{M}\left(e_{R}^{-} e_{L}^{+} \rightarrow \tilde{\nu}_{\alpha} \tilde{\bar{\nu}}_{\alpha}\right) & =\frac{e^{2} \beta s \sin \theta}{2 \sin ^{2} \theta_{W}}\left(\frac{\tan ^{2} \theta_{W}}{s-M_{Z}^{2}}\right) \quad(\alpha=e, \mu, \tau) .
\end{aligned}
$$

Here $\theta$ is the CM polar scattering angle, $m_{\chi_{i}^{+}}$are the chargino masses $(i=1$ is the lightest), while $\cos ^{2} \gamma_{R}$ is the mixing probability of the wino component in the lightest chargino; $\beta=\sqrt{1-4 \tilde{m}_{\nu}^{2} / s}$ is the sneutrino CM velocity, $s$ and $t$ are the usual invariant squares of energy and momentum transfer, $\theta_{W}$ is the weak angle. The differential cross section is then defined by $d \sigma / d \cos \theta=\Sigma|\mathcal{M}|^{2} \beta /(128 \pi s)$; we note that it contains an overall factor $\sin ^{2} \theta$, favoring wide angles with good experimental acceptance, well away from the beam-pipe. Figure 1 shows the integrated cross sections for energies $s=140,160,175,190 \mathrm{GeV}$, soon to be explored in successive upgrades of the LEP collider at CERN, for a range of $\tilde{m}_{\nu}$ values with $\cos ^{2} \gamma_{R} \simeq 1$ and $m_{\chi}=2 \tilde{m}_{\nu}$ (envisaging a 
scenario with the LSP mass $\tilde{m}_{\nu}$ close to the lightest neutralino mass $m_{\chi_{1}^{0}}$ and with the lightest chargino mass $m_{\chi_{1}^{+}} \simeq 2 m_{\chi_{1}^{0}}$ as in many models). We note that $\tilde{\bar{\nu}}_{e} \tilde{\nu}_{e}$ production is considerably enhanced above $\tilde{\bar{\nu}}_{\mu} \tilde{\nu}_{\mu}$ and $\tilde{\bar{\nu}}_{\tau} \tilde{\nu}_{\tau}$ production, due to the chargino-exchange contribution (with constructive interference). Adding all three flavors, Fig.1 indicates about $500(75)$ events per $100 p b^{-1}$ luminosity at $\sqrt{s}=190 \mathrm{GeV}$, for $\tilde{m}_{\nu}=50(80)$ $\mathrm{GeV}$, which should be enough to establish a clear signal in this mass range and possibly beyond, modulo detection efficiency and background questions discussed below.

If a sneutrino is the LSP, it can only decay by RPV. With the particle content of the minimal SUSY-SM (MSSM), the most general gauge- and SUSY-invariant Lagrangian includes the following terms that can mediate sneutrino decays [6]

$$
\mathcal{L}_{R P V}=\lambda_{i j k} L_{i} L_{j} E_{k}^{c}+\lambda_{i j k}^{\prime} L_{i} Q_{j} D_{k}^{c}
$$

where $L_{i}$ and $E_{i}^{c}$ are the (left-handed) lepton doublet and antilepton singlet chiral superfields (with generation index $i$ ), while $Q_{i}$ and $D_{i}^{c}$ are the quark doublet and charge- $1 / 3$ antiquark singlet superfields. Antisymmetry gives $\lambda_{i j k}=-\lambda_{j i k}$. In the MSSM these terms are all conventionally forbidden by a multiplicative symmetry called $R$-parity $\left(R_{p}\right)$, with $R_{p}=1$ for all SM particles and $R_{p}=-1$ for their SUSY partners, in order to prevent rapid proton decay. However, proton decay is forbidden if there are no additional $B$-violating terms, in which case either or both classes of $L$-violating terms above are allowed. Each such RPV term provides a possible decay channel into SM fermions as follows:

$$
\begin{aligned}
& \lambda_{i j k} \Rightarrow \tilde{\nu}_{i} \rightarrow \ell_{j R}^{+} \ell_{k R}^{-}, \quad \tilde{\nu}_{j} \rightarrow \ell_{i R}^{+} \ell_{k R}^{-}, \\
& \lambda_{i j k}^{\prime} \Rightarrow \tilde{\nu}_{i} \rightarrow \bar{d}_{j R} d_{k R}
\end{aligned}
$$

together with the charge-conjugate channels. The corresponding decay widths are therefore

$$
\Gamma\left(\tilde{\nu}_{i} \rightarrow \ell_{j}^{+} \ell_{k}^{-}\right)=\lambda_{i j k}^{2} \tilde{m}_{\nu} /(16 \pi), \quad \Gamma\left(\tilde{\nu}_{i} \rightarrow \bar{d}_{j} d_{k}\right)=3 \lambda_{i j k}^{\prime 2} \tilde{m}_{\nu} /(16 \pi)
$$

neglecting the lepton and quark masses. The requirement that $\tilde{\nu}$ decays within the detector (typically within $1 \mathrm{~m}$ ) translates into

$$
\lambda^{2}\left(\text { or } 3 \lambda^{\prime 2}\right) \gtrsim \beta \gamma\left(\mathrm{GeV} / \tilde{m}_{\nu}\right) \times 10^{-14}
$$

where $\beta \gamma=\sqrt{s /\left(4 \tilde{m}_{\nu}^{2}\right)-1}$ is the appropriate sneutrino Lorentz factor and $\lambda$ (or $\lambda^{\prime}$ ) denotes the coupling of the dominant decay process in Eq.(6) (or Eq.(17)). For the energies 
and masses of present interest, where Fig.1 shows appreciable cross sections, this implies very weak lower bounds $|\lambda|,\left|\lambda^{\prime}\right| \gtrsim 10^{-8}$ on the dominant couplings. We shall assume that all sneutrino flavors are near-degenerate and all decay directly by one of the RPV couplings above, neglecting $R_{p}$-conserving decays of heavier sneutrinos to the lightest sneutrino that are suppressed by phase-space and loop factors [but the latter decays would have very similar signatures anyway, with very little extra activity from the cascade process].

The alternative decay modes of Eqs.(6- -7) give quite distinctive signatures, that we now discuss.

(a) $L L E^{c}$-mediated decays: Eq.(6). Each sneutrino decays to two charged leptons, not necessarily of the same flavor; e.g. the coupling $\lambda_{121}$ would give

$$
\begin{aligned}
& \tilde{\nu}_{e} \rightarrow \mu^{+} e^{-}, \quad e^{+} e^{-} \rightarrow \tilde{\bar{\nu}}_{e} \tilde{\nu}_{e} \rightarrow\left(\mu^{-} e^{+}\right)\left(\mu^{+} e^{-}\right), \\
& \tilde{\nu}_{\mu} \rightarrow e^{+} e^{-}, \quad e^{+} e^{-} \rightarrow \tilde{\bar{\nu}}_{\mu} \tilde{\nu}_{\mu} \rightarrow\left(e^{-} e^{+}\right)\left(e^{+} e^{-}\right),
\end{aligned}
$$

Note that no invisible $\tilde{\nu}_{i} \rightarrow \nu_{j} \nu_{k}$ modes are accessed at tree level. Four-lepton final states like Eq.(10), with no missing energy and two invariant-mass constraints $m\left(\mu^{-} e^{+}\right)=$ $m\left(\mu^{+} e^{-}\right)=\tilde{m}_{\nu e}$ would be very striking and easily separated from SM backgrounds, that are small of order $\alpha^{4}$ and mostly contain low-mass QED pairs; the sum of $e^{+} e^{-} \rightarrow$ $Z Z \rightarrow(\ell \ell)(\ell \ell)$ backgrounds is $\lesssim 10^{-2} p b$. Final states like Eq.(11) would be similarly constrained, but with an ambiguity in the $e^{+} e^{-}$pairing to be resolved by mass matching. Analogous final states containing two $\tau$ leptons could be identified (including the $\tau^{ \pm}$charges) with good efficiency from the narrow few-prong $\tau$-decay topologies and/or displaced decay vertices. The directions of the tau momentum vectors would be approximately measurable and their magnitudes could be reconstructed from overall energy and momentum conservation [13]. Possible decays to four taus would also be recognizable and striking, but their reconstruction would be a zero-constraint fit with no protection against initial-state radiation corrections. In practice, in cases where the lepton pairing is ambiguous, one can select the pairing for which the two masses agree most closely and define their mean to be the best-fit sneutrino mass $\tilde{m}_{\nu}\left(\ell^{+} \ell^{-}\right)$.

(b) $L Q D^{c}$-mediated decays: Eq.(7). Each sneutrino decays to two charge- $1 / 3$ quarks, not necessarily of the same flavor, normally giving two jets. Flavor-tagging is possible for $b$-jets, but otherwise these modes are all essentially indistinguishable;

$$
\tilde{\nu}_{\alpha} \rightarrow j j, \quad e^{+} e^{-} \rightarrow \tilde{\bar{\nu}}_{\alpha} \tilde{\nu}_{\alpha} \rightarrow\left(j_{1} j_{2}\right)\left(j_{3} j_{4}\right),
$$


The 4-jet final state of Eq.(12) has no missing energy (except from semileptonic decays within b-jets) and two invariant-mass constraints $m\left(j_{1} j_{2}\right)=m\left(j_{3} j_{4}\right)=\tilde{m}_{\nu \alpha}$ to distinguish it from background. Of three possible dijet pairings, we should select the one where the dijet masses agree most closely, and define their mean to be the best-fit sneutrino mass $\tilde{m}_{\nu}(j j)$. QCD four-jet backgrounds are expected to be of order $\alpha_{s}^{2} \times \sigma\left(e^{+} e^{-} \rightarrow \bar{q} q\right) \sim 1$ pb, of which only a small fraction will accidentally satisfy the dijet mass constraint. Backgrounds from $e^{+} e^{-} \rightarrow W W \rightarrow j j j j$ are bigger, rising from about 1.5 to $9 \mathrm{pb}$ over the range $\sqrt{s}=160-190 \mathrm{GeV}$, but they obey their own dijet mass constraint $m\left(j_{1} j_{2}\right) \simeq m\left(j_{3} j_{4}\right) \simeq M_{W}$ that can be used to identify and remove most of these events; however, they will obscure any sneutrino signal with $\tilde{m}_{\nu}$ approaching $M_{W}$. It could be be advantageous to study decays like Eq.([2) at or below $\sqrt{s}=160 \mathrm{GeV}$ where the $W W$ background is relatively suppressed compared to lower-mass sneutrino signals. If the sneutrino signal happens to contain a $b$-jet, then $b$-tagging would also suppress the $W W$ background (though a fraction of the much smaller $e^{+} e^{-} \rightarrow Z Z$ background would survive).

(c) Mixed $L L E^{c} / L Q D^{c}$ decays. If the leading $L L E^{c}$ and $L Q D^{c}$ decay modes have comparable rates, there will be some events where one sneutrino decays to two leptons while the other decays to two jets, giving

$$
e^{+} e^{-} \rightarrow \tilde{\bar{\nu}}_{\alpha} \tilde{\nu}_{\alpha} \rightarrow\left(\ell^{+} \ell^{\prime}\right)\left(j_{1} j_{2}\right)
$$

with no missing energy (except sometimes with b-jets) and two invariant-mass constraints $m\left(\ell^{+} \ell^{\prime}\right)=m\left(j_{3} j_{4}\right)=\tilde{m}_{\nu \alpha}$ to distinguish them from backgrounds, that are small anyway (the sum of $e^{+} e^{-} \rightarrow Z Z \rightarrow(\ell \ell)(j j)$ backgrounds is $\lesssim 0.1 \mathrm{pb}$ ). Here $\ell$ and $\ell^{\prime}$ denote $e, \mu$, or $\tau$. Cases with different lepton flavors would be especially striking and background-free. Although dilepton and dijet masses should agree within resolution, the former usually gives a sharper estimate of $\tilde{m}_{\nu}$.

(d) Displaced vertices. If the sneutrino mean decay length is of order $0.1 \mathrm{~mm}-1 \mathrm{~m}$ (typically $\lambda \sim 10^{-6}-10^{-8}$ for cases of present interest), the two sneutrino decay vertices will usually be detectably displaced from each other and from the beam-intersection spot, providing an important extra signature (modulo some complications in events with final taus). This signature would discriminate strongly against most SM backgrounds, including $W W \rightarrow 4 j$.

To illustrate the invariant mass distributions, we impose typical gaussian resolution 
smearing on energies $E$, with $\Delta E=0.2 \sqrt{E / G e V}$ for leptons and $\Delta E=0.8 \sqrt{E / G e V}$ for jets. We also impose semi-realistic cuts, requiring all leptons and quarks to have rapidities $|\eta|<2$, energies $E>8 \mathrm{GeV}$, and angular separations $\theta_{i j}>20^{\circ}$. These cuts give about $80 \%$ acceptance for the examples we show. In the four-lepton and two-lepton-two-jet channels, there is little or no ambiguity about the pairings and the resulting best-fit sneutrino mass distributions have clean narrow peaks, that it is unnecessary to illustrate explicitly. The four-jet channels however have potentially serious $W W$ backgrounds. Figure 2 illustrates the distribution of best-fit sneutrino mass $\tilde{m}_{\nu}(j j)$ in four-jet cases like Eq.(12), for $\sqrt{s}=175 \mathrm{GeV}$ and $\tilde{m}_{\nu e}=50,65,80 \mathrm{GeV}$. The $W W$ background is calculated from the Pythia Monte Carlo, with a correction for missing neutrinos from semileptonic $c$-decays in addition to the energy smearing and acceptance cuts above. We see that the $\tilde{m}_{\nu}=50 \mathrm{GeV}$ mass peak is well above background. Assuming integrated luminosity $100 p b^{-1}$ and summing all three sneutrino flavors, the $\tilde{m}_{\nu}=65 \mathrm{GeV}$ case would predict a signal $S \simeq 100$ events in the $60-70 \mathrm{GeV}$ mass bin compared to a WW background $B \simeq 60$ events, giving significance $S / \sqrt{B}=13$. The $\tilde{m}_{\nu}=70 \mathrm{GeV}$ case would give only $S \simeq 60$ with higher $B \simeq 120$ and lower significance $S / \sqrt{B}=5$; this mass is about the limit for establishing a signal in the four-jet channel at $\sqrt{s}=175 \mathrm{GeV}$. Significance would be slightly improved with $\sqrt{s}=190 \mathrm{GeV}$ instead, but high luminosity may be harder to achieve here.

If a $\tilde{\nu}_{\mu} \rightarrow e^{+} e^{-}$or $\tilde{\nu}_{\tau} \rightarrow e^{+} e^{-}$decay signal were to be established (via $\lambda_{121}$ or $\lambda_{131}$ coupling), it would imply a corresponding resonance [7] in the $e^{+} e^{-} \rightarrow \tilde{\nu} \rightarrow e^{+} e^{-}$channel - or indeed for $e^{+} e^{-} \rightarrow \tilde{\nu} \rightarrow \mu^{+} \mu^{-}$if both $\lambda_{131}$ and $\lambda_{232}$ were significant. Such a resonance would have a large peak cross section of order $4 \pi / \tilde{m}_{\nu}^{2}$, four orders of magnitude above $\sigma_{Q E D}\left(e^{+} e^{-} \rightarrow \mu^{+} \mu^{-}\right)$, but a very narrow width; for the case $\tilde{m}_{\nu}=50 \mathrm{GeV}$, the upper limits[7] $\lambda_{121} \lesssim 0.04\left(m_{\tilde{e}} / 100 \mathrm{GeV}\right)$ and $\lambda_{131} \lesssim 0.1\left(m_{\tilde{e}} / 100 \mathrm{GeV}\right)$ indicate a width of 10 $\mathrm{MeV}$ at best, and it could be very much smaller. If these couplings were indeed near their upper limits above, such a resonance could be detected by a suitable scan; indeed, the absence of corrections to Bhabha scattering at TRISTAN already imposes significant further limits[] for $\tilde{m}_{\nu}=50-56 \mathrm{GeV}$. But for smaller couplings, $\lambda_{i j k}<10^{-3}$ say, the width would be less than $1 \mathrm{keV}$ giving five orders of magnitude suppression in a scanning bin of width $100 \mathrm{MeV}$, and the resonance signal would be lost. Similarly a hadronic decay signal $\tilde{\nu} \rightarrow \bar{q} q^{\prime}$ would imply the presence of $\bar{q} q^{\prime} \rightarrow \tilde{\nu} \rightarrow \bar{q} q^{\prime}$ resonance contributions 
in hadron collisions, but their narrow widths would make them much harder to detect than the SM $\bar{q} q \rightarrow Z \rightarrow \bar{q} q$ signals, which are themselves quite difficult to detect.

Finally, we note that hadron colliders too can put constraints on our sneutrino=LSP scenario, not only through direct electroweak production of sneutrinos but more importantly by hadroproduction of squarks and gluinos that would decay eventually to LSP pairs and hence to components like Eq.(6) in each final state. Since no anomalous four-lepton production has been reported at hadron colliders, it would appear either that $\tilde{\nu} \rightarrow e^{+} e^{-}, e^{ \pm} \mu^{\mp}, \mu^{+} \mu^{-}$are not dominant, or that squark and gluino production is strongly suppressed by high mass thresholds. On the other hand, decays $\tilde{\nu} \rightarrow e^{ \pm} \tau^{\mp}, \mu^{ \pm} \tau^{\mp}$ with at least one tau lepton are not disfavored and neither are analogous hadronic decays like Eq.(7).

Our results may be summarized as follows.

(1) We have shown that sneutrino pair production with RPV decays can give substantial and distinctive four-lepton or four-jet and possibly dilepton-dijet signals at the future upgraded LEP collider, if the LSP is a sneutrino and sneutrinos have approximately degenerate mass $\tilde{m}_{\nu} \lesssim 80 \mathrm{GeV}$.

(2) Production of $\tilde{\nu}_{e}$ pairs gives the biggest signals; $\tilde{\nu}_{\mu}$ and $\tilde{\nu}_{\tau}$ signals are similar but could have different lepton/jet flavors (if all sneutrinos decay directly via RPV) or could be essentially the same (if heavier sneutrinos decay first to the LSP, with negligible emission of soft particles). All sneutrino signals will generally be indistinguishable in the four-jet modes, in the absence of jet-flavor tagging.

(3) All signals have narrow dilepton and/or dijet invariant mass peaks at $m\left(\ell^{+} \ell^{-}\right), m(j j)=$ $\tilde{m}_{\nu}$, and may also possess displaced-decay-vertex signatures.

(4) The signals are cleanest in the four-lepton channels ( $L L E^{c}$-dominated decays), where there is little SM background. In the four-jet channel the most serious background appears to be $W W$ production, which is tolerable for $\tilde{m}_{\nu} \lesssim 70 \mathrm{GeV}$ but obliterates signals near $\tilde{m}_{\nu}=M_{W}$ (unless additional $b$-tag or displaced-vertex signatures are present).

(5) If a $\tilde{\nu}_{\mu} \rightarrow e^{+} e^{-}$or $\tilde{\nu}_{\tau} \rightarrow e^{+} e^{-}$decay signal were established, the corresponding $e^{+} e^{-} \rightarrow \tilde{\nu}$ resonance signals [7] could be detectable via a dedicated scan at lower energy, but only if its coupling $\lambda$ were not too far from the present upper limit. Possible $\bar{q} q^{\prime} \rightarrow \tilde{\nu} \rightarrow \bar{q} q^{\prime}$ signals would be unfeasible to detect at hadron colliders.

(6) The absence of reported $\ell_{1} \ell_{2} \ell_{3} \ell_{4}$ signals $\left(\ell_{i}=e, \mu\right)$ at hadron colliders, however, 
suggests that $\tilde{\nu} \rightarrow e e, e \mu, \mu \mu$ decays are unimportant, unless SUSY hadroproduction is suppressed by high mass thresholds.

\section{Acknowledgments}

We are grateful to Robert Sekulin for providing samples of $e^{+} e^{-} \rightarrow W W \rightarrow 4 j$ Monte Carlo events. RJNP thanks Gian Gopal for helpful conversations about LEP experimentation. This research was supported in part by the U.S. Department of Energy under Grants No. DE-FG02-95ER40896 and No. DE-FG02-84ER40173 and in part by the University of Wisconsin Research Committee with funds granted by the Wisconsin Alumni Research Foundation.

\section{References}

[1] For reviews see H.-P. Nilles, Phys. Rep. 110 (1984) 1; G.G. Ross, Grand Unified Theories, Benjamin Cummings (1985); H.E. Haber and G.L. Kane, Phys. Rep. 117 (1985) 75 .

[2] H. Baer, M. Drees and X. Tata, Phys. Rev. D41 (1990) 3414.

[3] The LEP collaborations ALEPH, DELPHI, L3 and OPAL, and the LEP Electroweak Working Group, CERN/PPE/94-187 (Nov.1994).

[4] D.O. Caldwell et al, Phys. Rev. Lett. 61 (1988) 510; SPP. Ahlen et al, Phys. Lett. B195 (1987) 603.

[5] N. Sato et al, Phys. Rev. D44 (1991) 2220.

[6] C.S. Aulakh and R.N. Mohapatra, Phys. Lett. B119, 316 (1982); L.J. Hall and M. Suzuki, Nucl. Phys. B231 (1984) 419; F. Zwirner, Phys. Lett. B132 (1983) 103; S. Dawson, Nucl. Phys. B261 (1985) 297. R. Barbieri and A. Masiero, Nucl. Phys. B267 (1986) 679; S. Dimopoulos and L.J. Hall, Phys. Lett. B207 (1987) 210; L. Hall, Mod. Phys. Lett. A5, 467 (1990); K.S. Babu and R.N. Mohapatra, Phys. Rev. D42 (1990) 3778.

[7] V. Barger, G.F. Giudice and T. Han, Phys. Rev. D40 (1989) 2987.

[8] K. Enqvist, A. Masiero and A. Riotto, Nucl. Phys. B373 (1992) 95; G. Bhattacharyya, J. Ellis, and K. Sridhar, CERN-TH.7530/94 (hep-ph/9503264); G. Bhattacharyya, D. Choudhury and K. Sridhar, CERN-TH-95-89 (hep-ph/9504314). 
[9] H. Dreiner and G.G. Ross, Nucl. Phys. B365 (1991) 597; H. Dreiner and R.J.N. Phillips, Nucl. Phys. B367 (1991) 591; V. Barger et al., Phys. Rev. D50, 4299 (1994); H. Baer, C. Kao and X. Tata, Phys. Rev. D51 (1995) 2180.

[10] H. Dreiner and S. Lola, Proc. of the Munich-Annecy-Hamburg Workshop on $e^{+} e^{-}$ collisions at $500 \mathrm{GeV}$ (ed. P.M. Zerwas), DESY 92-123B, p.707; L. Roszkowski, Waikaloa Workshop talk, hep-ph/9309208; R. Godbole, P. Roy, and X. Tata, Nucl. Phys. B401, 67 (1993); V. Barger, W.-Y. Keung and R.J.N. Phillips, hepph/9506321.

[11] see e.g. V. Barger, M.S. Berger and P. Ohmann, Phys. Rev. D49 4908 (1994).

[12] J. McCurry, Oxford thesis (1993).

[13] ALEPH collaboration, D. Decamp et al., Phys. Lett. B265 (1991) 475; DELPHI collaboration, P. Abreu et al., ibid B245 (1990) 276; L3 collaboration, B. Adeva et al., ibid B251 (1990) 311; OPAL collaboration, M.Z. Akrawy et al., Z. Phys. C49 (1991) 1 .

\section{Figures}

1. Integrated cross sections $\sigma\left(e^{+} e^{-} \rightarrow \tilde{\bar{\nu}}_{\alpha} \tilde{\nu}_{\alpha}\right)$ in pb versus sneutrino mass $\tilde{m}_{\nu}$ for a range of LEP energies and different sneutrino flavors. For $\tilde{\nu}_{e}$ pair production we assume the lightest chargino has mass $m_{\chi}=2 \tilde{m}_{\nu}$ and mixing $\cos ^{2} \gamma_{R} \simeq 1$.

2. Best-fit dijet sneutrino mass distributions for $\tilde{\nu}_{e}$ production at $\sqrt{s}=175 \mathrm{GeV}$ with $\tilde{m}_{\nu e}=50,65,80 \mathrm{GeV}$ and four-jet final states like Eq.(12), after the resolution smearing and acceptance cuts described in the text. The principal background from $W W \rightarrow 4 j$ is shown for comparison. 


$$
\sigma\left(e^{-} e^{+} \rightarrow \tilde{\nu}_{\alpha} \tilde{\nu}_{\alpha}\right)(\mathrm{pb})
$$

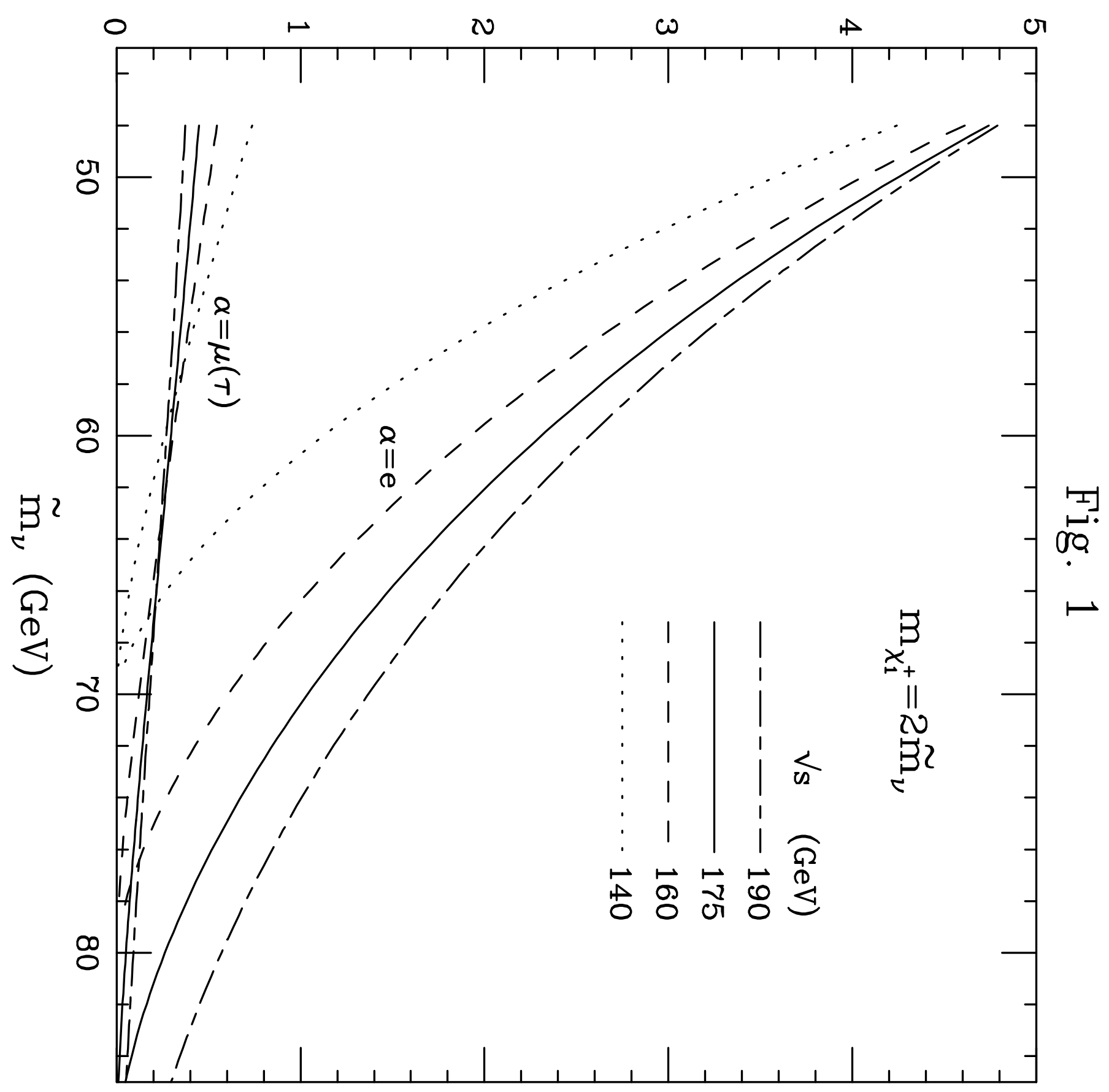




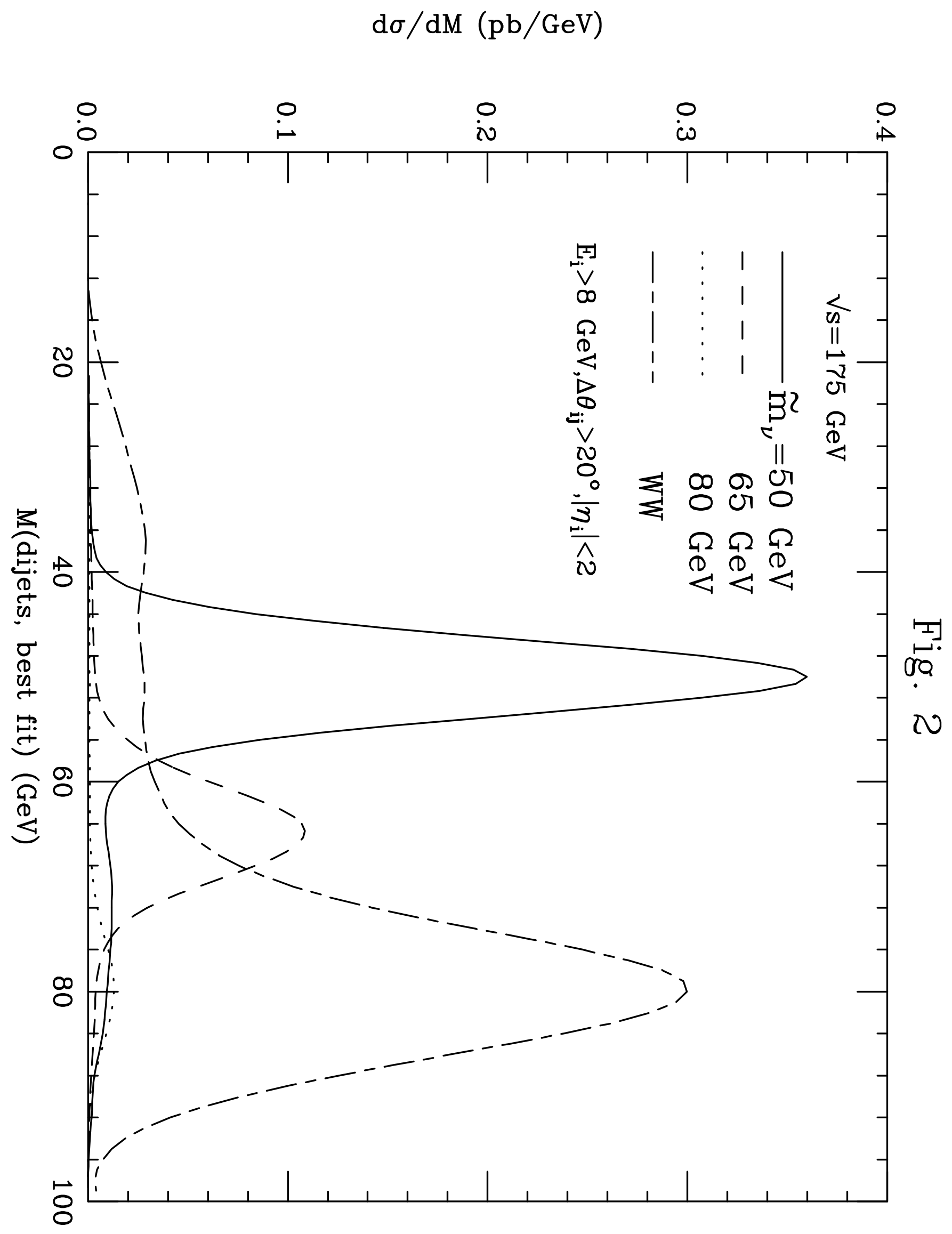

